\title{
Mouse Models in Prostate Cancer Translational Research: From Xenograft to PDX
}

\author{
Domenica Rea, ${ }^{1}$ Vitale del Vecchio, ${ }^{1}$ Giuseppe Palma, ${ }^{1}$ Antonio Barbieri, ${ }^{1}$ Michela Falco, \\ Antonio Luciano, ${ }^{1}$ Davide De Biase, ${ }^{2}$ Sisto Perdonà, ${ }^{3}$ Gaetano Facchini, ${ }^{4}$ and Claudio Arra ${ }^{1}$ \\ ${ }^{1}$ Animal Facility Unit, Department of Experimental Oncology, Istituto Nazionale per lo Studio e la Cura dei \\ Tumori "Fondazione G. Pascale", IRCCS, 80131 Naples, Italy \\ ${ }^{2}$ Department of Veterinary Medicine and Animal Productions, Università di Napoli Federico II, Via Delpino 1, 80137 Naples, Italy \\ ${ }^{3}$ Department of Urology, Istituto Nazionale per lo Studio e la Cura dei Tumori “Fondazione G. Pascale”, IRCCS, 80131 Naples, Italy \\ ${ }^{4}$ Division of Medical Oncology, Department of Uro-Gynaecological Oncology, Istituto Nazionale per lo Studio e la Cura dei \\ Tumori "Fondazione G. Pascale", IRCCS, 80131 Naples, Italy
}

Correspondence should be addressed to Domenica Rea; d.rea@istitutotumori.na.it

Received 17 March 2016; Accepted 21 April 2016

Academic Editor: Oreste Gualillo

Copyright (C) 2016 Domenica Rea et al. This is an open access article distributed under the Creative Commons Attribution License, which permits unrestricted use, distribution, and reproduction in any medium, provided the original work is properly cited.

\begin{abstract}
Despite the advancement of clinical and preclinical research on PCa, which resulted in the last five years in a decrement of disease incidence by $3-4 \%$, it remains the most frequent cancer in men and the second for mortality rate. Based on this evidence we present a brief dissertation on numerous preclinical models, comparing their advantages and disadvantages; among this we report the PDX mouse models that show greater fidelity to the disease, in terms of histopathologic features of implanted tumor, gene and miRNA expression, and metastatic pattern, well describing all tumor progression stages; this characteristic encourages the translation of preclinical results. These models become particularly useful in meeting the need of new treatments identification that eradicate PCa bone metastases growing, clarifying pathway of angiogenesis, identifying castration-resistant stem-like cells, and studying the antiandrogen therapies. Also of considerable interest are the studies of 3D cell cultures derived from PDX, which have the ability to maintain PDX cell viability with continued native androgen receptor expression, also showing a differential sensitivity to drugs. 3D PDX PCa may represent a diagnostic platform for the rapid assessment of drugs and push personalized medicine. Today the development of preclinical models in vitro and in vivo is necessary in order to obtain increasingly reliable answers before reaching phase III of the drug discovery.
\end{abstract}

\section{Introduction}

Despite the many scientific advances in pharmacological, clinical, and preclinical settings, prostate cancer (PCa) remains to be the first most common cancer in men [1]; it represents a major cause of cancer-related morbidity and mortality $[2,3]$. In the United States there were estimated 233,000 (27\%) new cancer prostate cases diagnosed in 2014, with an incidence of death valued at $29,480(10 \%)$ patients annually [4]. Currently the radical prostatectomy is one of the gold standards for the treatment of $\mathrm{PCa}$ [5], despite the recent pharmacological approach with novel antineoplastic developed and approved drugs (enzalutamide and abiraterone) that target the androgen receptor axis $[6,7]$, and also immunologic therapies with antiangiogenic molecules used in patients with progression of disease [8]. The lethal cases generally have a high Gleason score and can be metastatic and/or refractory to androgen deprivation therapy (castration-resistant prostate cancer mCRPC); these have a short survival of 1-3 years, depending on context. Skeletal metastasis is the most significant cause of morbidity and mortality in PCa; it is found in approximately $90 \%$ of patients who die because of PCa [9]; this one indicates that the bone microenvironment may promote the growth of PCa cells. The majority of bone lesions in $\mathrm{PCa}$ usually show a greater proportion of active osteoblasts than active osteoclasts, resulting in the net formation of bone [10]. On the other hand, despite the many new therapies for patients with advanced CRPC, 
the overall survival is still relatively short [11] due to an endocrine therapy resistance development in a part of patients. These mechanisms include interference of androgen receptor (AR) axis and inhibition of androgens biosynthesis $[1,12,13]$. Malignant cells derived from prostate epithelial layers of PCa, which include secretory luminal, basal, and rare neuroendocrine cells, lend to disease a high grade of heterogeneity [9]. A question is pending about which type of epithelial cell represents the origin of PCa: luminal stem cells, basal stem cells, or both [14]; this point still remains unclear, but it is likely that there is a complex explanation of the heterogeneity of the disease and the many genetic pathways that are involved [15]. Despite the incidence rate trend in $\mathrm{PCa}$, which continues to fall by $3-4 \%$ each year, and a large amount of prostate cancer studies, only very few findings have influenced the clinical management of the disease.

Innovative mouse models of prostate cancer have been developed to overcome the well-known limits and difficulties in PCa research [4]. For instance, the heterotopic models show some advantages like an easier tumor cells inoculation and in vivo tumor growth monitoring. While the heterotopic models mimic human prostate cancer in a more realistic way, the orthotopic implantation of tumor cells in the host more likely resembles the different tumor stages, dealing with native environment of tumor cells [16]. Independently from the advantages of these models in preclinical research, most studies are additionally hampered by a lack of standardization. Especially in orthotopic tumor cell application, the quality and number of inoculated tumor cells, as well as the addition of extracellular factors, have never been investigated in detail.

A major limitation in prostate cancer research is the lack of relevant preclinical models, which allow studying the molecular mechanisms of tumorigenesis. In fact, advanced in vitro and in vivo models are an indispensable requirement for the development of effective prevention and therapeutic intervention strategies [3]. To address and overcome the limitations of traditional models, reaching a greater loyalty compared to PCa human, currently patient-derived models (PDX) are used for preclinical research. Their use permits highlighting various aspects of PCa biology including angiogenesis, the identification of resistant castrate stem-like cells, and the effect of antiandrogen therapies [17]. PDX models are generated by using tumor tissues surgically removed from patient and plugged directly into the immune-compromised mice, without any manipulation in vitro. The tumors are subsequently maintained in vivo by mouse-to-mouse passages. Therefore we expect that PDX tumors models, which remain biologically stable and retain much of molecular, genetic, and histological characteristics, heterogeneity of original tumor, and response to treatment, become largely used in studies; instead, given the high costs of animal maintenance, lengthy latency period following engraftment, variable engraftment rates, and rare access to patient tissue specimens, PDX in vivo models are generally not yet widely employed in cancer research [18-20]. Mouse models can answer many questions about the etiology of cancer and help in screening new drugs and the development of more effective treatments. This work aims to collect and summarize data from research studies in order to identify, evaluate, and critically analyse in vivo models of PCa. Our ultimate goal was to obtain a systematic treatise about the progress as well as the advantages and disadvantages of these models in preclinical translational research.

\section{Prostate Cancer Mouse Models}

To date, preclinical prostate cancer mouse models still represent an essential tool to improve our understanding of PCa development, progression, and metastatic pattern. Spontaneous PCa transformation in different mouse strains is really uncommon [21]; therefore in the last years there have been created different generations of new manipulated mouse models of PCa to simulate all the stages of pathology, from the hyperplasia to HGPIN (high-grade prostatic intraepithelial neoplasia), until metastasis dissemination [21]; these transformation steps are slightly different from a histopathological point of view between human and mouse [22].

Xenograft mouse models (Table 1) represent a common "recipient" of human PCa, generated through orthotopic or heterotopic implantation of human tumor tissues, cell lines, or primary cell cultures, in nude mice [23], SCID [24], NODSCID [25], NOG/NSG [26], or RAG [27]. Different human prostate cancer cell lines have been used to perform various xenograft mouse models that, in this way, exhibit different features of the prostatic neoplasia. For instance LNCaP-LN3 cells, derived from androgen receptor mutated LNCaP [28], promote a high regional lymph node metastatic pattern after prostatic implantation, with castration-resistance features [29]. Instead PC3 cell lines, derived from bone metastasis of human PCa, are androgen-independent [30]; the PC-3M variant shows a higher ability to produce regional lymph node and distant organ metastasis [29].

Allograft mouse models (Table 1) represent a mouse tumor system that can be generated by using tumor cell lines/tissues derived from the same genetic background. In this way transplanted material and the host share the strain. TRAMP-C1/2/ $3[31,32]$ prostate cancer cell lines, derived from the prostatic epithelium of TRAMP mouse, and PTEN-CaP8 [33], from Pten-null transgenic mouse model of $\mathrm{PCa}$, are both in strain C57BL/6; instead PNEC30, originated from neuroendocrine cell population in mouse prostate cancer, is in strain BALB/c [34].

Genetically engineered mouse models (GEMMs) (Table 2) reproduce in depth the different stages of $\mathrm{PCa}$ associated with typical human genetic mutation that allow tumor progression. We recognize two generations of PCa GEMMs, the first one is characterized by the ectopic expression of Simian virus 40 (SV40) Large Antigen T (Tag) in prostate; this effector acts as an oncoprotein that negatively regulates p53 [35, 36]. In 1994 the C3(1)-Tag model was generated, the first GEMM under the expression of SV40 large Tag; this model showed prostatic epithelial hyperplasia about 3 months of age and local adenocarcinoma by 7-11 months of age [37]. Also the TRAMP model (transgenic adenocarcinoma of the mouse prostate) is based on the SV40 Tag activity, under the prostate-specific rat Probasin promoter [38]; these mice develop epithelial hyperplasia, after 8 weeks of age, PIN 
TABLE 1: Prostatic mouse models of engraftment.

\begin{tabular}{lccll}
\hline Type & $\begin{array}{c}\text { Background } \\
\text { strain }\end{array}$ & Year & Features & Disadvantages \\
\hline Xenograft & $\begin{array}{l}\text { Nude, SCID, } \\
\text { NOD-SCID, } \\
\text { NOG/NSG, RAG }\end{array}$ & 1996-2006 & $\begin{array}{l}\text { High take rate, low costs, LNCaP, } \\
\text { LNCaP-LN3, PC-3, PC-3M cell lines and } \\
\text { human prostatic tissues implanted }\end{array}$ & $\begin{array}{l}\text { Immune-compromised } \\
\text { mice tested }\end{array}$ \\
\hline Allograft & C57BL/6 & 1997-1994 & $\begin{array}{l}\text { High take rate, low costs, use of } \\
\text { immune-competent mice; } \\
\text { TRAMP-C1/2/3, PTEN-CaP8 cell lines } \\
\text { implanted }\end{array}$ & Low translational potential [31, 32, 38] \\
\hline Allograft & BALBC/c & 2004 & $\begin{array}{l}\text { High take rate, low costs, use of } \\
\text { immune-competent mice; use of PNEC30 } \\
\text { cell lines }\end{array}$ & $\begin{array}{l}\text { Low translational potential } \\
\text { cells }\end{array}$ \\
\hline
\end{tabular}

TABLE 2: Genetically engineered mouse models (GEMM) of prostate cancer.

\begin{tabular}{|c|c|c|c|c|c|}
\hline Name & Background strain & Year & Features & Disadvantages & Reference \\
\hline C3(1)-Tag & $\mathrm{FVB} / \mathrm{N}$ & 1994 & $\begin{array}{l}\text { PHH (3 mths) } \\
\text { PCa (7-11 mths) }\end{array}$ & $\begin{array}{l}\text { Sporadic metastases, aspecific } \\
\text { breast cancer in female (12 wks) }\end{array}$ & {$[37]$} \\
\hline TRAMP & $\mathrm{C} 57 \mathrm{BL} / 6$ & 1994 & $\begin{array}{l}\text { PHH (8 wks), PIN (18 wks) } \\
\text { Lymph. metastases ( } 28 \text { wks) }\end{array}$ & $\begin{array}{l}\text { Neuroendocrine originated } \\
\text { tumors }\end{array}$ & {$[38]$} \\
\hline FG-Tag & $\mathrm{C} 57 \mathrm{BL} / 6$ & 1996 & $\begin{array}{l}\text { Accurate model of } \\
\text { castration-resistant } \mathrm{PCa}\end{array}$ & $\begin{array}{l}\text { Aspecific adrenocortical tumors } \\
\text { in } 50 \% \text { of female mice }\end{array}$ & {$[39]$} \\
\hline LPB-Tag (LADY) & $\mathrm{CD}-1$ & 1997 & $\begin{array}{l}\text { Accurate model for all stages of } \\
\text { PCa studies }\end{array}$ & $\begin{array}{l}\text { Neuroendocrine originated foci } \\
\text { in liver, lymph., and lung } \\
\text { metastases }\end{array}$ & {$[40]$} \\
\hline $\begin{array}{l}\text { LPB-Tag/ARR } 2 \text { PB } \\
\text { hepsin }\end{array}$ & C57BL/6JxCBA & 2004 & Increased migratory ability & $\begin{array}{l}\text { Neuroendocrine originated cells } \\
\text { forming liver metastases }\end{array}$ & {$[41]$} \\
\hline Mt-PRL & $\mathrm{C} 57 \mathrm{BL} / 6 \mathrm{JxCBA}-\mathrm{f} 2$ & 1997 & Appropriate model to study $\mathrm{BPH}$ & $\begin{array}{l}\text { Rare PCa progression, no } \\
\text { metastases }\end{array}$ & {$[42]$} \\
\hline BK5-IGF1 & $\mathrm{FVB} \times \mathrm{ICR}$ & 2000 & PIN (6 mths), PCa (9 mths) & Not metastases, off-target effects & {$[43]$} \\
\hline PB-mAR & FVB & 2001 & $\begin{array}{l}\text { Microinvasive HGPIN, useful in } \\
\text { PCa studies about androgens }\end{array}$ & Rare metastases & {$[44]$} \\
\hline $\mathrm{ARR}_{2} \mathrm{~PB}-\mathrm{Myc}$ & FVB & 1999 & $\begin{array}{l}\text { From PIN to PCa in } 2 \mathrm{mths} \\
\text { Regression after castration } \\
(8 \mathrm{mths})\end{array}$ & Not metastases observed & {$[45]$} \\
\hline $\mathrm{ARR}_{2} \mathrm{~PB}-\mathrm{FGFR} 1$ & FVB & 2003 & Hyperproliferation and PIN & $\begin{array}{l}\text { Failed in the later stages of } \\
\text { disease }\end{array}$ & {$[46]$} \\
\hline PB-Ras & $\mathrm{FVB} / \mathrm{N}$ & 2004 & $\begin{array}{l}\text { Did not progress further than } \\
\text { PIN }\end{array}$ & $\begin{array}{l}\text { Intestinal metaplasia } \\
\text { Thickened fibromuscular stroma }\end{array}$ & {$[47]$} \\
\hline PB-Neu & C57BL/6JxCBA & 2006 & Similar to human acinar type & $\begin{array}{l}\text { Low translational potential (rare } \\
\text { cases of Neu alteration in human } \\
\text { PCa) }\end{array}$ & {$[48]$} \\
\hline $\mathrm{ARR}_{2} \mathrm{~PB}-\mathrm{ERG}$ & FVB & 2008 & PIN (12-14 wks) & Take rate of $38 \%$ & {$[49]$} \\
\hline
\end{tabular}

PHH: prostatic epithelial hyperplasia; PIN: prostatic intraepithelial neoplasia; BPH: benign prostatic hyperplasia.

(prostatic intraepithelial neoplasia) by 18 weeks, and lymph node metastasis by 28 weeks; this model has been the first that showed distant organ metastasis. The FG-Tag (fetal globin- $\gamma /$ T-antigen) [39], LPB-Tag (LADY) [40], and LPBTag/ARR ${ }_{2}$ PB-hepsin [41] chronologically represent the last example of first generation GEMM with a typical neuroendocrine cell transformation.

The second-generation GEMMs were born in 1997 with the Mt-PRL [42], a mouse model created to further study the role of prolactin (PRL) in prostate hyperplasia; the PRL ectopic expression has been inducted by Metallothionein-1
(Mt-1) promoter. Subsequently the BK5-IGF1 mouse models [43] were created which overexpressed the insulin-like growth factor 1, a molecule that resulted as an indicator of PCa in some patients that showed normal levels of PSA [62]. From 2001 there have been developed some other models using the Probasin $(\mathrm{PB})$ promoter, among which are the following: PB-mAR [44], to further understand the role of androgen receptor on the disease progression; $\mathrm{ARR}_{2} \mathrm{~PB}-\mathrm{Myc}$ that shows high levels of Myc, with a PIN progression and then invasive carcinoma, by the third month of age [45]; $\mathrm{ARR}_{2} \mathrm{~PB}-\mathrm{FGFR} 1$ models that show the physiological role of 
Fibroblast growth factor receptors 1 and 2 (FGFR1-2) that become upregulated in $40 \%$ of poorly differentiated $\mathrm{PCa}$ [46]; PB-Ras [47], PB-Neu [48], and $\mathrm{ARR}_{2}$ PB-ERG [49] instead were generated inducing an overexpression of any oncogenes that have an important role in different human tumor disease, as Neu in breast cancer [63] or Ras in any other [64].

Knockout models (Table 3 ) have been carried out by a different strategy that led to induce silencing of any tumor suppressor genes through deletion of whole sequences or part of them, essential for their activities. In 1993 RAR $\gamma$, the first $\mathrm{KO}$ mouse model of prostate cancer, was born; it was not able to develop PCa but only squamous metaplasia in mouse prostate and seminal vesicles [50]. Kip1 knockout mouse instead was generated through the loss of p27 [51]; this model showed hyperplasia of some organs among which is prostate. For these reasons it represents a very good model of BPH (benign prostatic hyperplasia). In 1999 Nkx3.1 KO model [52] that showed several prostatic phenotype as hyperplasia and dysplasia was generated but no tumor lesions have been detected. Finally we report the Pten KO, probably the best model to study PIN progression [53], without metastatic proliferation. The Pten-null background has been intercrossed with other $\mathrm{KO}$ to generate a more confident mouse model of PCa, for instance, Pten-null $\times$ p 27 [54] that showed the prostatic adenocarcinoma features or Pten-null $\times$ p53 that instead displays HGPIN [55]. Different conditional knockout models of PCa have been developed, during last years, using the Cre-loxP recombination system to excise specific DNA regions in somatic cells, through the gene regulation by tissue specific promoter as Probasin or PSA. Also these models are generated by specific gene silencing of PTEN [56], pRB [57], p53 [58], Apc [59], IGF-1 [60], and Brca2 [61].

\section{Prostate Tumor PDX Mouse Models}

Preclinical prostate cancer mouse models described so far fail to reproduce with high fidelity the different stages of tumorigenesis and the progression of disease observed in the clinic. Normally the xenograft cell lines lose the original tissue architecture of the site of origin, with related impaired physical and biochemical pattern of interaction with the environment, different gene expression, and altered response to pharmacological treatments $[65,66]$. Because of the issues described so far, PDX seems to be a good preclinical model to reproduce tumor features, maintaining more similarities; in this way many studies have shown the quality of PDX models highlighting histological compatibilities [67, 68], saved tumor cells heterogeneity [69], similar gene expression [70], gene variants [71], and miRNA pattern [72]. On the contrary there are no other limitations that suggest why these models are not still widely used, such as the more difficult tissue implantation procedures, especially in orthotopic mouse models [73, 74]; the site of implantation choice, in order to provide a good vascularization and cytokines/growth factors supply [73]; an average graft latency period from two to twelve months [75]; an average value of $23-75 \%$ of engraftment rates, strictly dependent on tumor aggressiveness [70]. We found studies that demonstrate a strong correlation between poor diagnosis and engraftment rates [68]. More authors suggest making some different serial engraftments $(<10)$ to increase the rates of mouse models, preserving the genetic integrity of parental tumor [76].

The human prostatic tumor xenotransplantation has been firstly described by Gittes in 1980 that reported high take rates $(\mathrm{TR}=50 \%)$ with the maintenance of many histological human tumor features in athymic nude mice [77]. Subsequently other studies showed a discouraging $\mathrm{TR}=0$ $2 \%$ obtained using moderate/well-differentiated carcinomas [78] that poorly increase transplanting pelvic lymph node metastatic tumors supplemented with T-pellets $(\mathrm{TR}=10 \%)$ [23]; all of the experiments described so far have carried out choosing, as a preferred strategy, the subcutaneous way of implantation, on the mice flank. Reid et al. have increased the TR in their studies, until hundred percent, treating mice with sheep or rabbit anti-mouse interferon serum (globulin fraction) [79]. These latter results have shown that the use of athymic nude mice did not improve the procedure and so the authors decided to change the immunodeficient mice strain, now choosing the NMRI or CB17-NOD athymic nude mice which have a reduced NK activity; Wang et al. [80] described a $\mathrm{TR}=58,1 \%$ using these strains also supplemented with $\mathrm{T}$ pellet, but in this work the authors decide to investigate also the other way of implantation: subrenal capsule $(\mathrm{TR}=93,4 \%)$ and orthotopical ( $\mathrm{TR}=71,9 \%)$.

From a histological point of view Gittes [77] showed for the first time a study that demonstrated the development of squamous metaplasia and interstitial fibrosis in the PCa SC xenograft mouse models. They underlined any different modifications in the insert, from the parental carcinoma. Subsequently, for many different tumors, the high cytohistopathological similarity before and after the transplantation has been demonstrated $[79,81,82]$; in addition, both of them had the same Gleason score [83]. Moreover other studies also reported a substantial matching for the PAP and PSA expression in serum, as biomarkers [84, 85]. In most of this work also the metastatic pattern of the different models has been studied in depth; for instance, Hoehn et al. [84] did not find metastasis in any organs during necropsy in PC-82 tumor heterotransplant. On the other hand Lubaroff et al. [86] reported lung metastasis in SCID mice.

According to all of the reported studies so far, it can be affirmed that currently the subrenal capsule (SRC) represents the best way of transplantation because of the high TR; this site represents the gold standard thanks to a high vascularization, a good interstitial fluid pressure, and a high lymphatic flow; those ensure an even nutrients, hormones, oxygen, and growth factors supply [87]; however this site permits the easy placement of an insert with right size [88]. Lin et al. described the SRC grafting procedure using small cutting tumor pieces $\left(1 \times 3 \times 3 \mathrm{~mm}^{3}\right.$ in size $)$ implanted in male NOD/SCID and supplemented with testosterone. After 3 to 6 months of growth the animals were sacrificed and the masses were harvested and reimplanted in SRC. The xenograft pieces were maintained for up to 3 years, increasing the aggressiveness during each passage. Subsequently, in the last passage, the hosts were sacrificed and examined for 


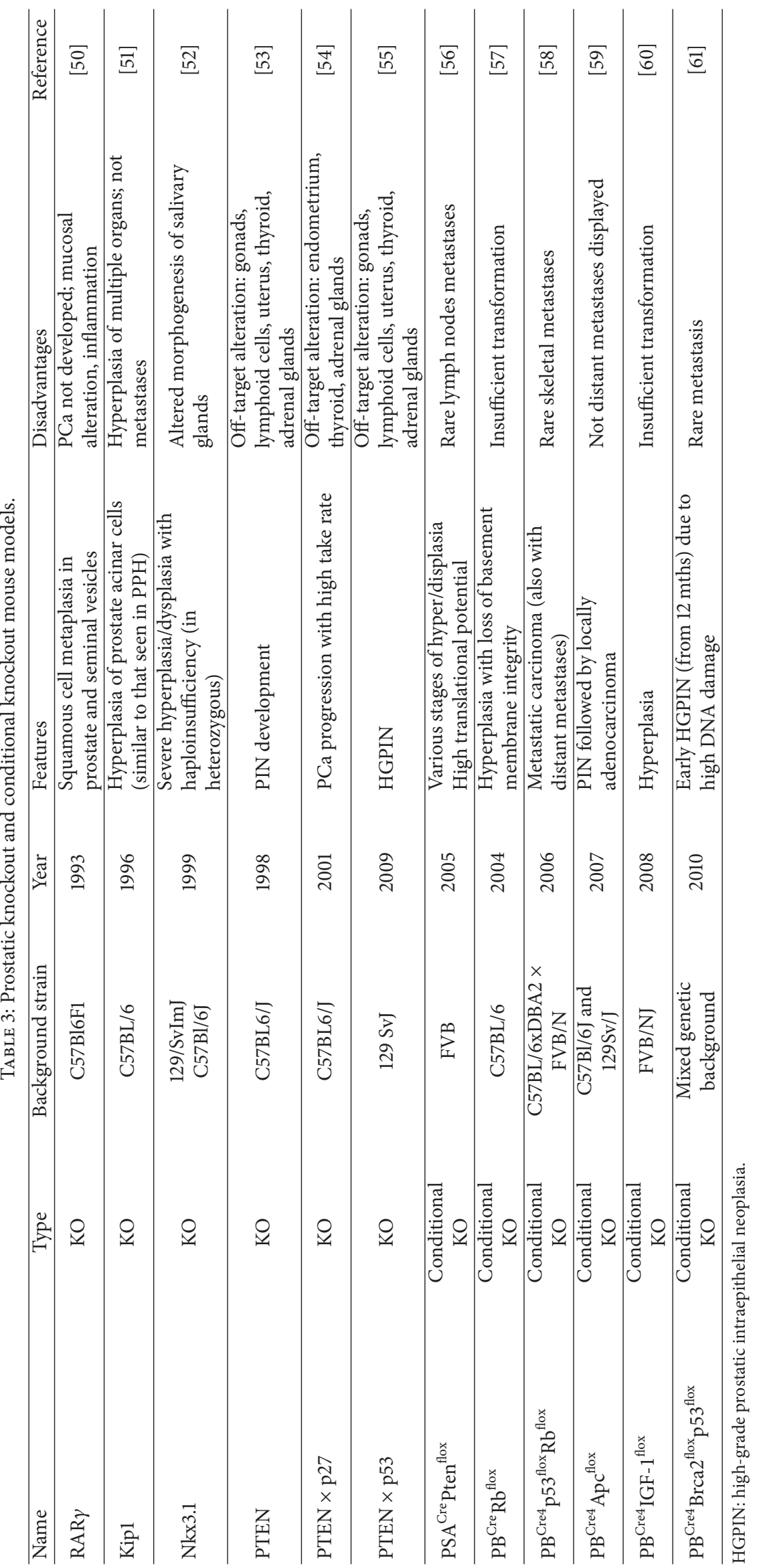


metastasis in lymph nodes, lungs, liver, kidneys, spleen, and femur [89].

This procedure allows preserving the tumor cells heterogeneity; in fact different studies, focused on prostatic cancer but also on many other different tumors, have shown that during the first phases of xenotransplantation the various tumor cells subpopulation are subjected to an anoxia status caused by the total absence of vascularization that make a selective pressure on them and only the most resistant cells variant can tolerate this environment; the SRC implantation attenuates these hard conditions. This statements have been supported by different studies that confirmed the high similarity between SRC xenograft and the parent tumors in terms of androgen sensitivity, histopathology, biomarkers expression, and metastatic potential [90-93]. Furthermore it has been demonstrated that also the subcutaneous reengraftment, in NSG or NOD-SCID mice, is improved after a well SRC establishment of the insert (TR 95\%); this procedure is used to better control the tumor growth, therapeutic responsiveness, or metastatic potential [94].

Given this data we can consider the PDX mouse models like a powerful tool to study the biology of cancer from early stages of tumor progression, until the metastatic dissemination, without losing architectural tissue features or molecular and genetic basis of the disease.

\section{The "Big Things" in PCa Modelling: PDX 3D Spheroids and Humanized Mice}

To date the research on prostate cancer has been hindered not only by the lack of relevant tumor models for clinical use, but also by the heterogeneity of cancer patients. During the last years many studies have investigated the use of PDX derived cell lines to generate disease models; in particular the use of 3D cellular culture was favored with respect to using the 2D monolayer cell lines. 3D spheroids more closely resemble in vivo tissue in terms of cellular communication and the development of extracellular matrices, while standard 2D cell cultures are inadequate representations of this environment, which often makes them unreliable predictors of in vivo drug efficacy and toxicity [18, 95-97]. These evidences reveal that the cell stimulation by environment represents a crucial feature to generate a better disease model; for instance, the bone metastatic PCa cells, whether derived from cell lines or primary PDX tumor tissue, show poor viability and the inability to grow in $2 \mathrm{D}$ culture; this is an established phenomenon that indicates the lack of critical components from the bone microenvironment upon which these highly adapted cells depend $[98,99]$. To overcome these critical issues, the PDX cancer cells were encapsulated within threedimensional hyaluronic acid-based hydrogels (HA); such a system has demonstrated that the hydrogel maintains the viability of the cells with the native PDX continuous expression of the androgen receptor [17]. The hydrogel encapsulation provides the means to fully recapitulate the tumor microenvironment with precise, tunable control over architectural and mechanical cues and/or critical cell-extracellular matrix interactions, specifically, as a ubiquitous component of the bone marrow where bone metastatic PCa cells reside. HA plays an active role in regulating several biological processes, including tumorigenesis, strongly justifying its use as an extracellular matrix analogue for culturing bone metastatic tumor cells in vitro [100]. Fong et al. showed that 3D PDX PCa cells exhibited an increased resistance to docetaxel as compared to a standard cell line commonly used in PCa research. The discoveries translation from mouse models to clinical trials has been hampered by the genetic differences between human and inbreed mouse strains and also by the inability to recapitulate human immunological system; for these reasons new and more performing humanized models are required; these tools represent useful ground-breaking platforms for cancer research. To generate humanized mice with functional human immune system, animals with severe immunodeficiency as NOD scid gamma $\left(\mathrm{NSG}^{\mathrm{TM}}\right)$ are used; they have a severe combined immune deficiency mutation (scid) and IL2 receptor gamma chain deficiency, resulting in a lack of mature T cells and B cells and impaired NK cells, and are deficient in cytokine signalling $[101,102]$. In the last few years, in order to eliminate the remaining adaptive and innate immunity in host mice, the animals are inoculated with Human Peripheral Blood Mononuclear Cells (PBMCs) or hematopoietic stem cells CD34+ (HSCs) to create a stable human immune system. PDX-engrafted humanized-NSG models represent the next step in cancer modelling, because they offer a unique platform to examine human $\mathrm{T}$ celldependent and B cell-dependent responses against clinically relevant tumors and to induce the immune system action versus specific tumor types. In PCa research these models can be considered as a highly validated instrument in human tumor microenvironment studies, also to test tumor response to both Standard Oncologic Cares treatments and novel compounds. They represent an affordable approach to validate single immune-based as well as preclinical combination therapies and allowing new individualized cancer treatments $[102,103]$.

\section{Conclusion}

Major limitation in prostate cancer research is the lack of relevant preclinical models, which allow studying the molecular mechanisms of tumorigenesis. In fact, advanced in vitro and in vivo models represent an indispensable requirement for the development of new therapeutic strategies. Currently there is a continued research for innovative $\mathrm{PCa}$ in vivo models with greater fidelity to disease, which try to foster the translation of preclinical findings into the clinic, particularly to satisfy the need to identify new treatments that will eradicate PCa metastases growing in bone.

The xenograft models represent a great tool to study the mechanisms underlying many human tumors, including efficacy of specific treatments, or cancer stem cell-like properties.

Nevertheless they show some limits related to a compromised immune system of the host that instead becomes crucial in human $\mathrm{PCa}$, especially in metastatic dissemination pattern. In addition, using stabilized or primary tumor cell line, in xenograft, the complete bypassing of all the tumor initiation stages as the interaction with microenvironment or angiogenesis process has been observed. Using cell lines 
derived from human metastasis, also the metastatic development was impaired [104].

The allograft mouse models results are widely used because they overcome the limits represented by the use of an immunosuppressed host; in this way it is possible to study in depth the interaction between tumor progression and immune system; on the other hand they do not show any limitations linked with the use of not human biological materials.

The GEMMs have been allowed to reproduce the similar genetic alterations that occur in human. So we can divide all the transgenic mouse models discussed in two different categories, on the basis of typical features. The firstgeneration GEMMs showed an aggressive phenotype, often with metastatic proliferation, castration-resistant $\mathrm{PCa}$, due to the SV40 Tag action on p53 and pRB (not present in human), and a high incidence of neuroendocrine aberrant transformation. Instead the second generation, created through ectopic expression of endogenous oncogenic effectors, seems to not be able to reproduce the different human disease stages of PCa in mouse.

The last generation of preclinical mouse models is represented by PDXs that offer a powerful means for studying biological pathways in cancer and for testing new drugs. Given the data reported, they seem to closely reproduce the progression of $\mathrm{PCa}$, representing a better predictive model compared with all those that are generated by established or primary cell line transplantation, further tracking the various passages of tumor progression, from implantation to metastatic dissemination. Instead, especially in PCa models, we have described the different issues that occur during the procedures, correlated with the choice of the site of implantation; this leads to a less broad availability of prostatic tumor PDX models than others, as breast cancer PDX, that can be developed through transplantation in different sites: interscapular or mammary fat pad and renal capsule.

To date many scientists have developed different tools to overcome the limits of the PDXs; one of the most promising ones seems to be the in vitro PDX 3D cell cultures. This procedure considers all the PDX passages, with the advantage of reducing the number of animal hosts [17], subsequently transferring all the experimental conditions in 3D cell cultures. This technology represents a valid preclinical model that bypasses the issues of 2D monolayer cell cultures; it may be used for a rapid and high throughput platform to assess drug efficacy and to find new predictive biomarkers for novel targeted therapies [91].

The models discussed here have considerable importance to understand the tumor pathogenesis and the complex biology of PCa. They also represent a promising support to enhance development of new approaches to prevention, detection, and treatment of this malignancy. These tools will be useful for a better and faster pharmacological screening in drug discovery and personalized medicine. Furthermore, PDX mouse models will allow a more profound knowledge about a still unexplored area of research in preclinical mouse models generation such as the interaction between human xenogenic stroma and the neoplasm. In the near future, new models for translational research are expected to be generated; they will aim to ameliorate the correlation between results obtained in animal models and human patients. Given the data it could be necessary to generate new preclinical mouse models to enhance understanding of PCa development and progression to metastasis.

\section{Competing Interests}

The authors confirm that this paper's content has no potential competing interests.

\section{Authors' Contributions}

Domenica Rea and Vitale del Vecchio contributed equally and are co-first authors.

\section{Acknowledgments}

This work was supported by Italian Ministry of Health RF2010-2316780. The authors would like to express their gratitude to Mr. Massimiliano Spinelli, Data Manager of Animal Facility Unit, Department of Experimental Oncology, Istituto Nazionale per lo Studio e la Cura dei Tumori "Fondazione G. Pascale", IRCCS, Naples, Italy, for kind help in providing informatic assistance.

\section{References}

[1] A. R. Mehta and A. J. Armstrong, "Tasquinimod in the treatment of castrate-resistant prostate cancer-current status and future prospects," Therapeutic Advances in Urology, vol. 8, no. 1, pp. 9-18, 2016.

[2] A. Jemal, R. Siegel, E. Ward et al., "Cancer statistics, 2008," CA Cancer Journal for Clinicians, vol. 58, no. 2, pp. 71-96, 2008.

[3] M. Saar, C. Körbel, V. Jung et al., "Experimental orthotopic prostate tumor in nude mice: techniques for local cell inoculation and three-dimensional ultrasound monitoring," Urologic Oncology: Seminars and Original Investigations, vol. 30, no. 3, pp. 330-338, 2012.

[4] R. Siegel, J. Ma, Z. Zou, and A. Jemal, "Cancer statistics, 2014," CA: A Cancer Journal for Clinicians, vol. 64, no. 1, pp. 9-29, 2014.

[5] Y. I. Lee, H. M. Lee, J. K. Jo et al., "Association between seminal vesicle invasion and prostate cancer detection location after transrectal systemic biopsy among men who underwent radical prostatectomy," PLoS One, vol. 11, no. 2, article e0148690, 2016.

[6] T. M. Beer, A. J. Armstrong, D. E. Rathkopf et al., "Enzalutamide in metastatic prostate cancer before chemotherapy," The New England Journal of Medicine, vol. 371, no. 5, pp. 424-433, 2014.

[7] J. S. de Bono, C. J. Logothetis, A. Molina et al., "Abiraterone and increased survival in metastatic prostate cancer," The New England Journal of Medicine, vol. 364, no. 21, pp. 1995-2005, 2011.

[8] G. R. Thoreson, B. A. Gayed, P. H. Chung, and G. V. Raj, "Emerging therapies in castration resistant prostate cancer," The Canadian Journal of Urology, vol. 21, no. 2, supplement 1, pp. 98 105, 2014.

[9] K. C. Valkenburg and B. O. Williams, "Mouse models of prostate cancer," Prostate Cancer, vol. 2011, Article ID 895238, 22 pages, 2011. 
[10] J. Dai, C. L. Hall, J. Escara-Wilke, A. Mizokami, J. M. Keller, and E. T. Keller, "Prostate cancer induces bone metastasis through Wnt-induced bone morphogenetic protein-dependent and independent mechanisms," Cancer Research, vol. 68, no. 14, pp. 5785-5794, 2008.

[11] V. A. DiPippo, H. M. Nguyen, L. G. Brown, W. C. Olson, R. L. Vessella, and E. Corey, "Addition of PSMA ADC to enzalutamide therapy significantly improves survival in in vivo model of castration resistant prostate cancer," The Prostate, vol. 76, no. 3, pp. 325-334, 2016.

[12] K. Chi, S. J. Hotte, A. M. Joshua et al., "Treatment of mCRPC in the AR-axis-targeted therapy-resistant state," Annals of Oncology, vol. 26, no. 10, Article ID mdv267, pp. 2044-2056, 2015.

[13] Y. N. S. Wong, R. Ferraldeschi, G. Attard, and J. De Bono, "Evolution of androgen receptor targeted therapy for advanced prostate cancer," Nature Reviews Clinical Oncology, vol. 11, no. 6, pp. 365-376, 2014.

[14] X. Wang, M. K.-D. Julio, K. D. Economides et al., "A luminal epithelial stem cell that is a cell of origin for prostate cancer," Nature, vol. 461, no. 7263, pp. 495-500, 2009.

[15] G. P. Risbridger and R. A. Taylor, "The complexities of identifying a cell of origin for human prostate cancer," Asian Journal of Andrology, vol. 13, no. 1, pp. 118-119, 2011.

[16] K. J. Pienta, C. Abate-Shen, D. B. Agus et al., "The current state of preclinical prostate cancer animal models," Prostate, vol. 68, no. 6, pp. 629-639, 2008.

[17] E. L. S. Fong, M. Martinez, J. Yang et al., "Hydrogel-based 3D model of patient-derived prostate xenograft tumors suitable for drug screening," Molecular Pharmaceutics, vol. 11, no. 7, pp. 2040-2050, 2014.

[18] J. J. Tentler, A. C. Tan, C. D. Weekes et al., "Patient-derived tumour xenografts as models for oncology drug development," Nature Reviews Clinical Oncology, vol. 9, no. 6, pp. 338-350, 2012.

[19] D. Siolas and G. J. Hannon, "Patient-derived tumor xenografts: transforming clinical samples into mouse models," Cancer Research, vol. 73, no. 17, pp. 5315-5319, 2013.

[20] R. Kirk, "Prostate cancer from basal cells," Nature Reviews Clinical Oncology, vol. 7, article 550, 2010.

[21] S. B. Shappell, G. V. Thomas, R. L. Roberts et al., "Prostate pathology of genetically engineered mice: definitions and classification. the consensus report from the bar harbor meeting of the mouse models of human cancer consortium prostate pathology committee," Cancer Research, vol. 64, no. 6, pp. 2270-2305, 2004.

[22] R. A. DePinho, “The age of cancer," Nature, vol. 408, no. 6809, pp. 248-254, 2000.

[23] W. M. van Weerden, C. M. A. De Ridder, C. L. Verdaasdonk et al., "Development of seven new human prostate tumor xenograft models and their histopathological characterization," The American Journal of Pathology, vol. 149, no. 3, pp. 1055-1062, 1996.

[24] N. Craft, Y. Shostak, M. Carey, and C. L. Sawyers, "A mechanism for hormone-independent prostate cancer through modulation of androgen receptor signaling by the HER-2/neu tyrosine kinase," Nature Medicine, vol. 5, no. 3, pp. 280-285, 1999.

[25] J. A. Nemeth, J. F. Harb, U. Barroso Jr., Z. He, D. J. Grignon, and M. L. Cher, "Severe combined immunodeficient-hu model of human prostate cancer metastasis to human bone," Cancer Research, vol. 59, no. 8, pp. 1987-1993, 1999.
[26] J. M. D’Antonio, D. J. van der Griend, L. Antony et al., "Loss of androgen receptor-dependent growth suppression by prostate cancer cells can occur independently from acquiring oncogenic addiction to androgen receptor signaling," PLoS ONE, vol. 5, no. 7, Article ID el1475, 2010.

[27] Q. Zhang, X. J. Yang, S. D. Kundu et al., "Blockade of transforming growth factor- $\beta$ signaling in tumor-reactive $\mathrm{CD}^{+} \mathrm{T}$ cells activates the antitumor immune response cycle," Molecular Cancer Therapeutics, vol. 5, no. 7, pp. 1733-1743, 2006.

[28] J. Veldscholte, C. Ris-Stalpers, G. G. J. M. Kuiper et al., "A mutation in the ligand binding domain of the androgen receptor of human INCaP cells affects steroid binding characteristics and response to anti-androgens," Biochemical and Biophysical Research Communications, vol. 173, no. 2, pp. 534-540, 1990.

[29] C. A. Pettaway, S. Pathak, G. Greene et al., "Selection of highly metastatic variants of different human prostatic carcinomas using orthotopic implantation in nude mice," Clinical Cancer Research, vol. 2, no. 9, pp. 1627-1636, 1996.

[30] M. E. Kaighn, K. S. Narayan, Y. Ohnuki, J. F. Lechner, and L. W. Jones, "Establishment and characterization of a human prostatic carcinoma cell line (PC-3)," Investigative Urology, vol. 17, no. 1, pp. 16-23, 1979.

[31] B. A. Foster, J. R. Gingrich, E. D. Kwon, C. Madias, and N. M. Greenberg, "Characterization of prostatic epithelial cell lines derived from transgenic adenocarcinoma of the mouse prostate (TRAMP) model," Cancer Research, vol. 57, no. 16, pp. 33253330, 1997.

[32] N. M. Greenberg, F. DeMayo, M. J. Finegold et al., "Prostate cancer in a transgenic mouse," Proceedings of the National Academy of Sciences of the United States of America, vol. 92, no. 8, pp. 3439-3443, 1995.

[33] J. Jiao, S. Wang, R. Qiao et al., "Murine cell lines derived from Pten null prostate cancer show the critical role of PTEN in hormone refractory prostate cancer development," Cancer Research, vol. 67, no. 13, pp. 6083-6091, 2007.

[34] Y. Hu, T. Wang, G. D. Stormo, and J. I. Gordon, "RNA interference of achaete-scute homolog 1 in mouse prostate neuroendocrine cells reveals its gene targets and DNA binding sites," Proceedings of the National Academy of Sciences of the United States of America, vol. 101, no. 15, pp. 5559-5564, 2004.

[35] D. P. Lane and L. V. Crawford, "T antigen is bound to a host protein in SV40-transformed cells," Nature, vol. 278, no. 5701, pp. 261-263, 1979.

[36] J. A. DeCaprio, J. W. Ludlow, J. Figge et al., "SV40 large tumor antigen forms a specific complex with the product of the retinoblastoma susceptibility gene," Cell, vol. 54, no. 2, pp. 275283, 1988.

[37] I. G. Maroulakou, M. Anver, L. Garrett, and J. E. Green, "Prostate and mammary adenocarcinoma in transgenic mice carrying a rat C3(1) simian virus 40 large tumor antigen fusion gene," Proceedings of the National Academy of Sciences of the United States of America, vol. 91, no. 23, pp. 11236-11240, 1994.

[38] N. M. Greenberg, F. J. DeMayo, P. C. Sheppard et al., "The rat probasin gene promoter directs hormonally and developmentally regulated expression of a heterologous gene specifically to the prostate in transgenic mice," Molecular Endocrinology, vol. 8, no. 2, pp. 230-239, 1994.

[39] C. Perez-Stable, N. H. Altman, J. Brown, M. Harbison, C. Cray, and B. A. Roos, "Prostate, adrenocortical, and brown adipose tumors in fetal globin/T antigen transgenic mice," Laboratory Investigation, vol. 74, no. 2, pp. 363-373, 1996. 
[40] Y. Yan, P. C. Sheppard, S. Kasper et al., "Large fragment of the probasin promoter targets high levels of transgene expression to the prostate of transgenic mice," Prostate, vol. 32, no. 2, pp. 129-139, 1997.

[41] O. Klezovitch, J. Chevillet, J. Mirosevich, R. L. Roberts, R. J. Matusik, and V. Vasioukhin, "Hepsin promotes prostate cancer progression and metastasis," Cancer Cell, vol. 6, no. 2, pp. 185195,2004

[42] H. Wennbo, J. Kindblom, O. G. P. Ísaksson, and J. Törnell, "Transgenic mice overexpressing the prolactin gene develop dramatic enlargement of the prostate gland," Endocrinology, vol. 138, no. 10, pp. 4410-4415, 1997.

[43] J. DiGiovanni, K. Kiguchi, A. Frijhoff et al., "Deregulated expression of insulin-like growth factor 1 in prostate epithelium leads to neoplasia in transgenic mice," Proceedings of the National Academy of Sciences of the United States of America, vol. 97, no. 7, pp. 3455-3460, 2000.

[44] M. Stanbrough, I. Leav, P. W. L. Kwan, G. J. Bubley, and S. P. Balk, "Prostatic intraepithelial neoplasia in mice expressing an androgen receptor transgene in prostate epithelium," Proceedings of the National Academy of Sciences of the United States of America, vol. 98, no. 19, pp. 10823-10828, 2001.

[45] G. Jenster, "The role of the androgen receptor in the development and progression of prostate cancer," Seminars in Oncology, vol. 26, no. 4, pp. 407-421, 1999.

[46] K. W. Freeman, B. E. Welm, R. D. Gangula et al., "Inducible prostate intraepithelial neoplasia with reversible hyperplasia in conditional FGFR1-expressing mice," Cancer Research, vol. 63, no. 23, pp. 8256-8263, 2003.

[47] A. Scherl, J.-F. Li, R. D. Cardiff, and N. Schreiber-Agus, "Prostatic intraepithelial neoplasia and intestinal metaplasia in prostates of Probasin-RAS transgenic mice," Prostate, vol. 59, no. 4, pp. 448-459, 2004.

[48] Z. Li, M. Szabolcs, J. D. Terwilliger, and A. Efstratiadis, "Prostatic intraepithelial neoplasia and adenocarcinoma in mice expressing a probasin-Neu oncogenic transgene," Carcinogenesis, vol. 27, no. 5, pp. 1054-1067, 2006.

[49] S. A. Tomlins, B. Laxman, S. Varambally et al., "Role of the TMPRSS2-ERG gene fusion in prostate cancer," Neoplasia, vol. 10, no. 2, pp. 177-188, 2008.

[50] D. Lohnes, P. Kastner, A. Dierich, M. Mark, M. LeMeur, and P. Chambon, "Function of retinoic acid receptor $\gamma$ in the mouse," Cell, vol. 73, no. 4, pp. 643-658, 1993.

[51] K. Nakayama, N. Ishida, M. Shirane et al., "Mice lacking p $27^{\text {Kipl }}$ display increased body size, multiple organ hyperplasia, retinal dysplasia, and pituitary tumors," Cell, vol. 85, no. 5, pp. 707-720, 1996.

[52] R. Bhatia-Gaur, A. A. Donjacour, P. J. Sciavolino et al., "Roles for Nkx3.1 in prostate development and cancer," Genes and Development, vol. 13, no. 8, pp. 966-977, 1999.

[53] A. Di Cristofano, B. Pesce, C. Cordon-Cardo, and P. P. Pandolfi, "Pten is essential for embryonic development and tumour suppression," Nature Genetics, vol. 19, no. 4, pp. 348-355, 1998.

[54] C. Abate-Shen, W. A. Banach-Petrosky, X. Sun et al., "Nkx3.1; Pten mutant mice develop invasive prostate adenocarcinoma and lymph node metastases," Cancer Research, vol. 63, no. 14, pp. 3886-3890, 2003.

[55] S. S. Couto, M. Cao, P. C. Duarte et al., "Simultaneous haploinsufficiency of Pten and Trp53 tumor suppressor genes accelerates tumorigenesis in a mouse model of prostate cancer," Differentiation, vol. 77, no. 1, pp. 103-111, 2009.
[56] S. Wang, J. Gao, Q. Lei et al., "Prostate-specific deletion of the murine Pten tumor suppressor gene leads to metastatic prostate cancer," Cancer Cell, vol. 4, no. 3, pp. 209-221, 2003.

[57] L. A. Maddison, B. W. Sutherland, R. J. Barrios, and N. M. Greenberg, "Conditional deletion of $\mathrm{Rb}$ causes early stage prostate cancer," Cancer Research, vol. 64, no. 17, pp. 6018-6025, 2004.

[58] Z. Zhou, A. Flesken-Nikitin, D. C. Corney et al., "Synergy of p53 and $\mathrm{Rb}$ deficiency in a conditional mouse model for metastatic prostate cancer," Cancer Research, vol. 66, no. 16, pp. 7889-7898, 2006.

[59] K. J. Bruxvoort, H. M. Charbonneau, T. A. Giambernardi et al., "Inactivation of Apc in the mouse prostate causes prostate carcinoma," Cancer Research, vol. 67, no. 6, pp. 2490-2496, 2007.

[60] B. W. Sutherland, S. E. Knoblaugh, P. J. Kaplan-Lefko, F. Wang, M. Holzenberger, and N. M. Greenberg, "Conditional deletion of insulin-like growth factor-I receptor in prostate epithelium," Cancer Research, vol. 68, no. 9, pp. 3495-3504, 2008.

[61] J. C. Francis, A. McCarthy, M. K. Thomsen, A. Ashworth, and A. Swain, "Brca2 and Trp53 deficiency cooperate in the progression of mouse prostate tumourigenesis," PLoS Genetics, vol. 6, no. 6, pp. 1-9, 2010.

[62] J. M. Chan, M. J. Stampfer, E. Giovannucci et al., "Plasma insulin-like growth factor-I and prostate cancer risk: a prospective study," Science, vol. 279, no. 5350, pp. 563-566, 1998.

[63] T. Sorlie, C. M. Perou, R. Tibshirani et al., "Gene expression patterns of breast carcinomas distinguish tumor subclasses with clinical implications," Proceedings of the National Academy of Sciences of the United States of America, vol. 98, no. 19, pp. 1086910874, 2001.

[64] N. Shimizu, M. Ohtsubo, and S. Minoshima, "MutationView/ KMcancerDB: a database for cancer gene mutations," Cancer Science, vol. 98, no. 3, pp. 259-267, 2007.

[65] A. Goodspeed, L. M. Heiser, J. W. Gray, and J. C. Costello, "Tumor-derived cell lines as molecular models of cancer pharmacogenomics," Molecular Cancer Research, vol. 14, no. 1, pp. 3-13, 2016.

[66] S. Domcke, R. Sinha, D. A. Levine, C. Sander, and N. Schultz, "Evaluating cell lines as tumour models by comparison of genomic profiles," Nature Communications, vol. 4, article 2126, 2013.

[67] P. Loukopoulos, K. Kanetaka, M. Takamura, T. Shibata, M. Sakamoto, and S. Hirohashi, "Orthotopic transplantation models of pancreatic adenocarcinoma derived from cell lines and primary tumors and displaying varying metastatic activity," Pancreas, vol. 29, no. 3, pp. 193-203, 2004.

[68] Y. S. Derose, G. Wang, Y.-C. Lin et al., “Tumor grafts derived from women with breast cancer authentically reflect tumor pathology, growth, metastasis and disease outcomes," Nature Medicine, vol. 17, no. 11, pp. 1514-1520, 2011.

[69] C. C. Whiteford, S. Bilke, B. T. Greer et al., "Credentialing preclinical pediatric xenograft models using gene expression and tissue microarray analysis," Cancer Research, vol. 67, no. 1, pp. 32-40, 2007.

[70] X. Zhao, Z. Liu, L. Yu et al., "Global gene expression profiling confirms the molecular fidelity of primary tumor-based orthotopic xenograft mouse models of medulloblastoma," NeuroOncology, vol. 14, no. 5, pp. 574-583, 2012.

[71] C. L. Morton and P. J. Houghton, "Establishment of human tumor xenografts in immunodeficient mice," Nature Protocols, vol. 2, no. 2, pp. 247-250, 2007. 
[72] P. N. Bogner, S. K. Patnaik, R. Pitoniak et al., "Lung cancer xenografting alters microRNA profile but not immunophenotype," Biochemical and Biophysical Research Communications, vol. 386, no. 2, pp. 305-310, 2009.

[73] J. M. Fleming, T. C. Miller, M. J. Meyer, E. Ginsburg, and B. K. Vonderhaar, "Local regulation of human breast xenograft models," Journal of Cellular Physiology, vol. 224, no. 3, pp. 795806, 2010.

[74] J. E. Talmadge, R. K. Singh, I. J. Fidler, and A. Raz, "Murine models to evaluate novel and conventional therapeutic strategies for cancer," The American Journal of Pathology, vol. 170, no. 3, pp. 793-804, 2007.

[75] A. Bergamaschi, G. O. Hjortland, T. Triulzi et al., "Molecular profiling and characterization of luminal-like and basal-like in vivo breast cancer xenograft models," Molecular Oncology, vol. 3, no. 5-6, pp. 469-482, 2009.

[76] B. Rubio-Viqueira, A. Jimeno, G. Cusatis et al., "An in vivo platform for translational drug development in pancreatic cancer," Clinical Cancer Research, vol. 12, no. 15, pp. 4652-4661, 2006.

[77] R. F. Gittes, "The nude mouse-its use as tumor-bearing model of the prostate," Progress in Clinical and Biological Research, vol. 37, pp. 31-37, 1980.

[78] M. A. Jones, G. Williams, and A. J. S. Davies, "Value of xenografts in the investigation of prostatic function: preliminary communication," Journal of the Royal Society of Medicine, vol. 73, no. 10, pp. 708-712, 1980.

[79] L. M. Reid, N. Minato, I. Gresser, J. Holland, A. Kadish, and B. R. Bloom, "Influence of anti-mouse interferon serum on the growth and metastasis of tumor cells persistently infected with virus and of human prostatic tumors in athymic nude mice," Proceedings of the National Academy of Sciences of the United States of America, vol. 78, no. 2, pp. 1171-1175, 1981.

[80] Y. Wang, M. P. Revelo, D. Sudilovsky et al., "Development and characterization of efficient xenograft models for benign and malignant human prostate tissue," The Prostate, vol. 64, no. 2, pp. 149-159, 2005.

[81] Y. Shimosato, T. Kameya, K. Nagai et al., "Transplantation of human tumors in nude mice," Journal of the National Cancer Institute, vol. 56, no. 6, pp. 1251-1260, 1976.

[82] Z. Csapo, K. Brand, R. Walther, and K. Fokas, "Comparative experimental study of the serum prostate specific antigen and prostatic acid phosphatase in serially transplantable human prostatic carcinoma lines in nude mice," The Journal of Urology, vol. 140, no. 5, pp. 1032-1038, 1988.

[83] W. J. Huss, D. R. Gray, E. S. Werdin, W. K. Funkhouser Jr., and G. J. Smith, "Evidence of pluripotent human prostate stem cells in a human prostate primary xenograft model," Prostate, vol. 60, no. 2, pp. 77-90, 2004.

[84] W. Hoehn, F. H. Schroeder, J. F. Reimann, A. C. Joebsis, and P. Hermanek, "Human prostatic adenocarcinoma: some characteristics of a serially transplantable line in nude mice (PC 82)," The Prostate, vol. 1, no. 1, pp. 95-104, 1980.

[85] D. R. McCulloch, K. Opeskin, E. W. Thompson, and E. D. Williams, "BM18: a novel androgen-dependent human prostate cancer xenograft model derived from a bone metastasis," Prostate, vol. 65, no. 1, pp. 35-43, 2005.

[86] D. M. Lubaroff, M. B. Cohen, L. D. Shultz, and W. G. Beamer, "Survival of human prostate carcinoma, benign hyperplastic prostate tissues, and IL-2-activated lymphocytes in SCID mice," Prostate, vol. 27, no. 1, pp. 32-41, 1995.
[87] C. E. Ott and F. G. Knox, “Tissue pressures and fluid dynamics in the kidney," Federation Proceedings, vol. 35, no. 8, pp. 1872$1875,1976$.

[88] N. J. Robertson, P. J. Fairchild, and H. Waldmann, "Ectopic transplantation of tissues under the kidney capsule," Methods in Molecular Biology, vol. 380, pp. 347-353, 2007.

[89] D. Lin, A. W. Wyatt, H. Xue et al., "High fidelity patient-derived xenografts for accelerating prostate cancer discovery and drug development," Cancer Research, vol. 74, no. 4, pp. 1272-1283, 2014.

[90] C.-H. Lee, H. Xue, M. Sutcliffe et al., "Establishment of subrenal capsule xenografts of primary human ovarian tumors in SCID mice: potential models," Gynecologic Oncology, vol. 96, no. 1, pp. 48-55, 2005.

[91] J.-C. Cutz, J. Guan, J. Bayani et al., "Establishment in severe combined immunodeficiency mice of subrenal capsule xenografts and transplantable tumor lines from a variety of primary human lung cancers: potential models for studying tumor progression-related changes," Clinical Cancer Research, vol. 12, no. 13, pp. 4043-4054, 2006.

[92] X. Dong, J. Guan, J. C. English et al., "Patient-derived first generation xenografts of non-small cell lung cancers: promising tools for predicting drug responses for personalized chemotherapy," Clinical Cancer Research, vol. 16, no. 5, pp. 1442-1451, 2010.

[93] D. Lin, H. Xue, Y. Wang et al., "Next generation patient-derived prostate cancer xenograft models," Asian Journal of Andrology, vol. 16, no. 3, pp. 407-412, 2014.

[94] D. Lin, J. Bayani, Y. Wang et al., "Development of metastatic and non-metastatic tumor lines from a patient's prostate cancer specimen-identification of a small subpopulation with metastatic potential in the primary tumor," Prostate, vol. 70, no. 15, pp. 1636-1644, 2010.

[95] J. Kondo, H. Endo, H. Okuyama et al., "Retaining cell-cell contact enables preparation and culture of spheroids composed of pure primary cancer cells from colorectal cancer," Proceedings of the National Academy of Sciences of the United States of America, vol. 108, no. 15, pp. 6235-6240, 2011.

[96] V. C. Daniel, L. Marchionni, J. S. Hierman et al., "A primary xenograft model of small-cell lung cancer reveals irreversible changes in gene expression imposed by culture in vitro," Cancer Research, vol. 69, no. 8, pp. 3364-3373, 2009.

[97] M. D. Landis, B. D. Lehmann, J. A. Pietenpol, and J. C. Chang, "Patient-derived breast tumor xenografts facilitating personalized cancer therapy," Breast Cancer Research, vol. 15, no. 1, article 201, 2013.

[98] L. A. Gurski, A. K. Jha, C. Zhang, X. Jia, and M. C. FarachCarson, "Hyaluronic acid-based hydrogels as 3D matrices for in vitro evaluation of chemotherapeutic drugs using poorly adherent prostate cancer cells," Biomaterials, vol. 30 , no. 30, pp. 60766085, 2009.

[99] X. Xu, L. A. Gurski, C. Zhang, D. A. Harrington, M. C. FarachCarson, and X. Jia, "Recreating the tumor microenvironment in a bilayer, hyaluronic acid hydrogel construct for the growth of prostate cancer spheroids," Biomaterials, vol. 33, no. 35, pp. 9049-9060, 2012.

[100] E. Burdett, F. K. Kasper, A. G. Mikos, and J. A. Ludwig, "Engineering tumors: a tissue engineering perspective in cancer biology," Tissue Engineering Part B: Reviews, vol. 16, no. 3, pp. 351359, 2010.

[101] M. A. Brehm, N. Jouvet, D. L. Greiner, and L. D. Shultz, "Humanized mice for the study of infectious diseases," Current Opinion in Immunology, vol. 25, no. 4, pp. 428-435, 2013. 
[102] L. Gonzalez, N. Strbo, and E. R. Podack, "Humanized mice: novel model for studying mechanisms of human immunebased therapies," Immunologic Research, vol. 57, no. 1-3, pp. 326334, 2013.

[103] Q. Zhou, J. Facciponte, M. Jin, Q. Shen, and Q. Lin, "Humanized NOD-SCID IL2 $\mathrm{rg}^{-1-}$ mice as a preclinical model for cancer research and its potential use for individualized cancer therapies," Cancer Letters, vol. 344, no. 1, pp. 13-19, 2014.

[104] J. T. Buijs and G. van der Pluijm, "Osteotropic cancers: from primary tumor to bone," Cancer Letters, vol. 273, no. 2, pp. 177193, 2009. 


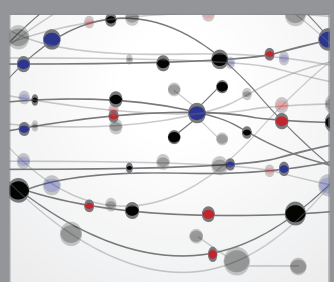

The Scientific World Journal
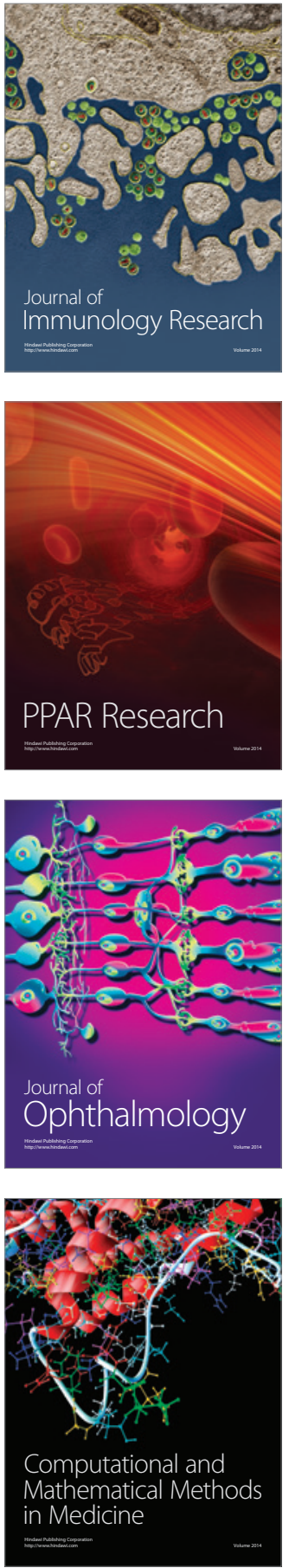

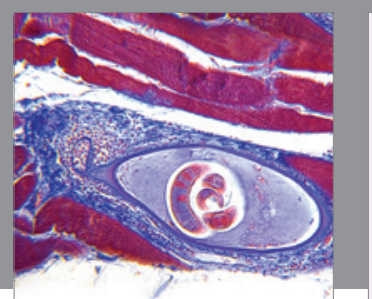

Gastroenterology Research and Practice

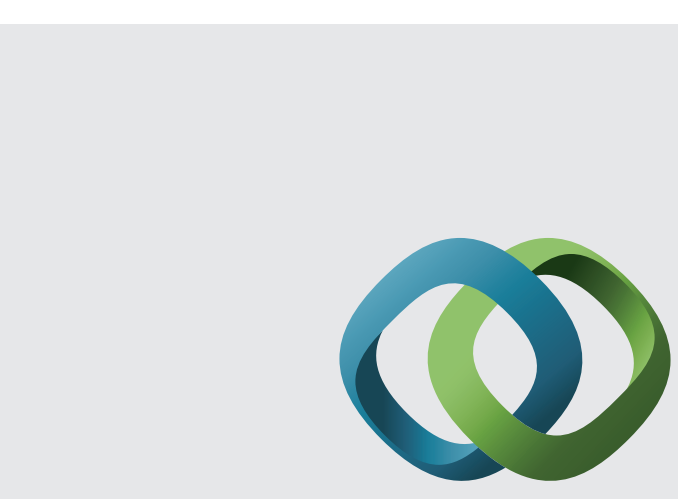

\section{Hindawi}

Submit your manuscripts at

http://www.hindawi.com
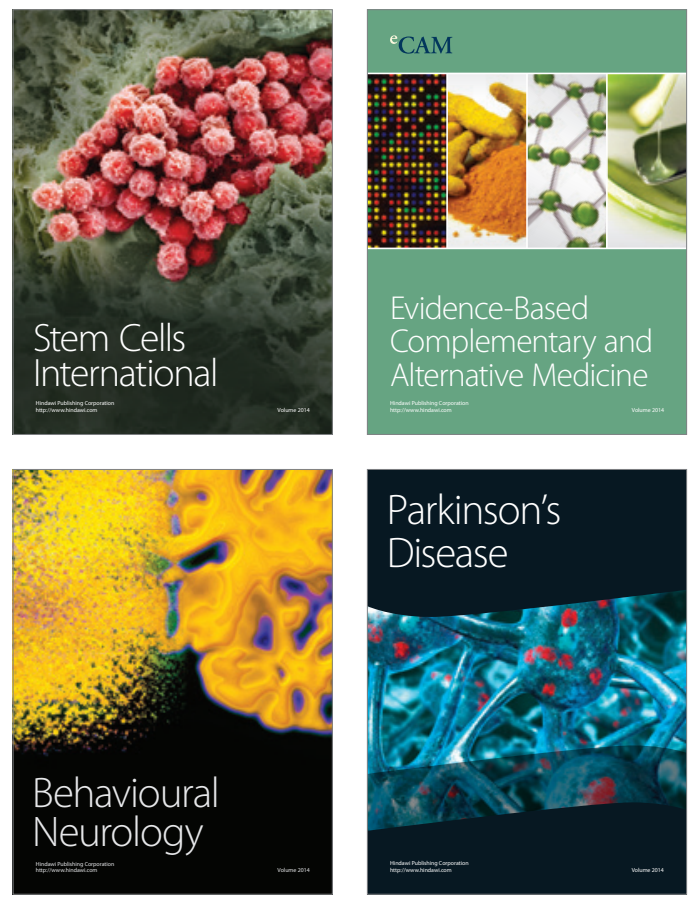
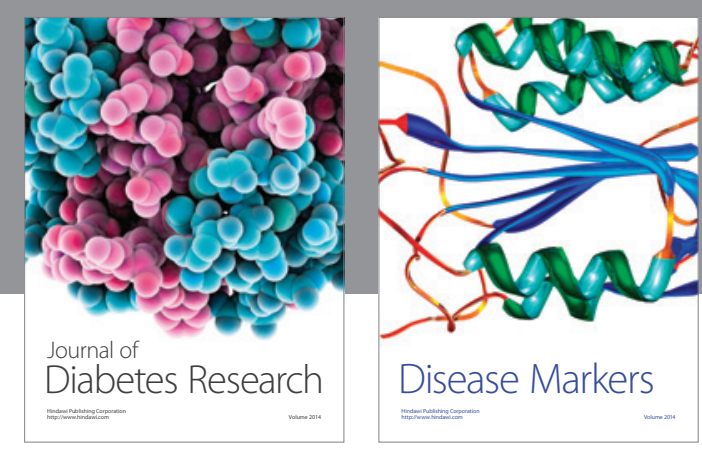

Disease Markers
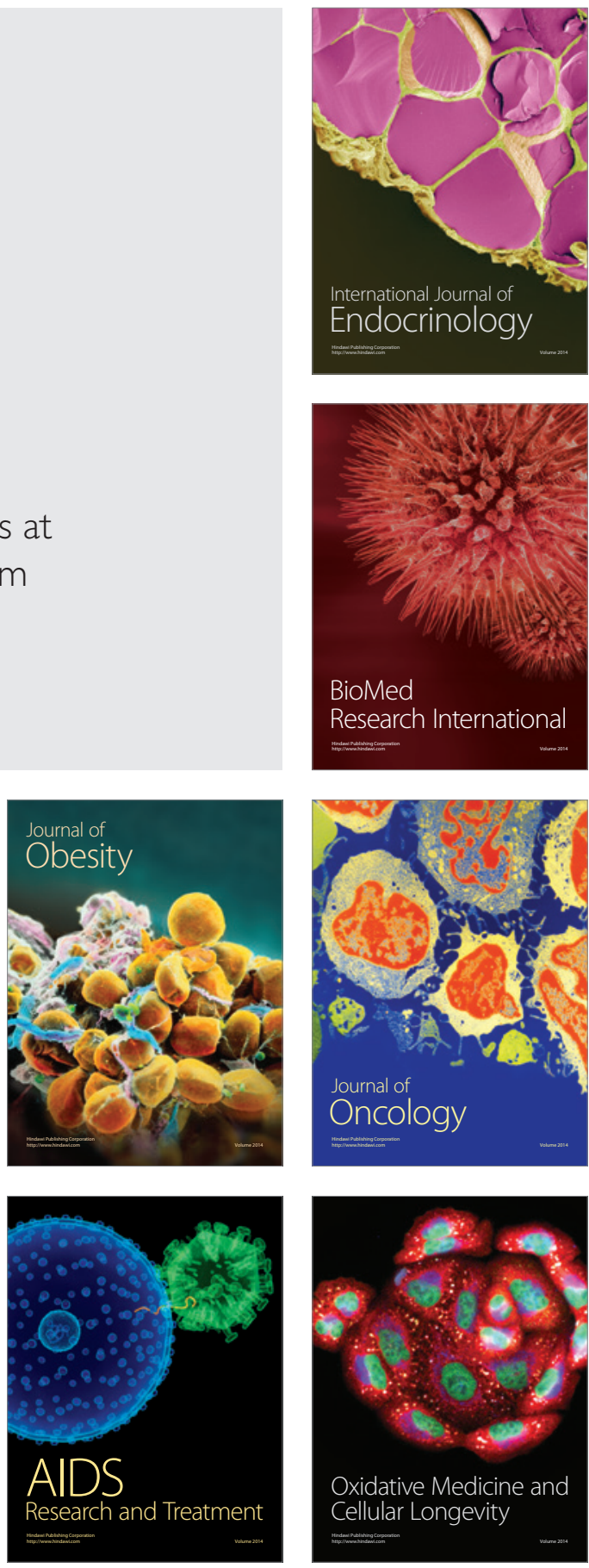